DRAFT VERSION OCTOBER 31, 2018

Preprint typeset using $\mathrm{LT}_{\mathrm{E}} \mathrm{X}$ style emulateapj v. 11/26/04

\title{
CONSTRAINING LOW-FREQUENCY ALFVÉNIC TURBULENCE IN THE SOLAR WIND USING DENSITY FLUCTUATION MEASUREMENTS
}

\author{
Benjamin D. G. Chandran ${ }^{1}$, Eliot Quataert ${ }^{2}$, Gregory G. Howes ${ }^{3}$, Qian Xia ${ }^{1}$, , PeEra Pongkitiwanichakul $^{1}$ \\ Draft version October 31, 2018
}

\begin{abstract}
One proposed mechanism for heating the solar wind, from close to the sun to beyond $\sim 10 \mathrm{AU}$, invokes lowfrequency, oblique, Alfvén-wave turbulence. Because small-scale oblique Alfvén waves (kinetic Alfvén waves) are compressive, the measured density fluctuations in the solar wind place an upper limit on the amplitude of kinetic Alfvén waves and hence an upper limit on the rate at which the solar wind can be heated by lowfrequency, Alfvénic turbulence. We evaluate this upper limit for both coronal holes at $5 R_{\odot}$ and in the nearEarth solar wind. At both radii, the upper limit we find is consistent with models in which the solar wind is heated by low-frequency Alfvénic turbulence. At $1 \mathrm{AU}$, the upper limit on the turbulent heating rate derived from the measured density fluctuations is within a factor of 2 of the measured solar wind heating rate. Thus if low-frequency Alfvénic turbulence contributes to heating the near-Earth solar wind, kinetic Alfvén waves must be one of the dominant sources of solar wind density fluctuations at frequencies $\sim 1 \mathrm{~Hz}$. We also present a simple argument for why density fluctuation measurements $d o$ appear to rule out models in which the solar wind is heated by non-turbulent high frequency waves "sweeping" through the ion-cyclotron resonance, but are compatible with heating by low-frequency Alfvénic turbulence.
\end{abstract}

Subject headings:

\section{INTRODUCTION}

A number of observations indicate that the solar wind undergoes spatially extended heating. For example, in situ measurements from satellites such as Helios \& Voyager show that electrons and protons have non-adiabatic temperature profiles at heliocentric distances $r \sim 0.3-50 \mathrm{AU}$ (e.g., Freeman 1988; Gazis et al. 1994; Richardson et al. 1995; Cranmer et al. 2009). Similarly, remote UVCS observations detect large ion temperatures that increase with heliocentric distance within a few solar radii of the Sun (Kohl et al 1998, Antonucci et al 2000). The observed heating likely plays a critical role in accelerating the solar wind to supersonic and super-Alfvénic speeds and also significantly impacts the plasma properties in the near-Earth space environment. ${ }^{4}$

Several heating mechanisms have been proposed to account for these observations, including magnetic reconnection (Fisk 2003; Fisk et al 2003), plasma instabilities driven by an electron heat flux or cross-field currents (Markovskii \& Hollweg 2002a, 2002b; Markovskii 2004), non-turbulent waves at frequencies comparable to the proton cyclotron frequency $\Omega_{i}$ (Abraham-Shrauner \& Feldman 1977, Hollweg \& Turner 1978, McKenzie et al 1979, Hollweg 1981, Tu \& Marsch 1997, Marsch \& Tu 1997, Hollweg \& Isenberg 2002), turbulent waves extending from low frequencies up $\sim \Omega_{i}$ (Isenberg \& Hollweg 1983, Tu et al 1984, Marsch 1991, Chandran 2005), and turbulence that fluctuates only on time scales

\footnotetext{
${ }^{1}$ Space Science Center and Department of Physics, University of New Hampshire, Durham, NH; benjamin.chandran@unh.edu, qdy2@cisunix.unh.edu, \& pbu3@cisunix.unh.edu

${ }^{2}$ Astronomy Department \& Theoretical Astrophysics Center, 601 Campbell Hall, The University of California, Berkeley, CA 94720; eliot@astro.berkeley.edu

${ }^{3}$ Department of Physics and Astronomy, University of Iowa, Iowa City, IA, USA; gregory-howes@uiowa.edu

${ }^{4}$ Some studies, however, dispute that the observed temperature profiles imply heating, claiming that many of the observed properties of the solar wind can be explained by strong heating localized at the coronal base and the collisionless propagation of non-thermal particle distributions from the corona out into the interplanetary medium (see, e.g., Scudder 1992ab).
}

$\gg \Omega_{i}^{-1}$. (Coleman 1968; Matthaeus et al 1999; Dmitruk et al 2001, 2002; Cranmer \& van Ballegooijen 2005; Cranmer, van Ballegooijen, \& Edgar 2007; Verdini \& Velli 2007; Chandran et al 2009). This paper focuses on this last class of models, those involving low-frequency turbulence.

These models hypothesize that Alfvén waves are launched into the corona by photospheric motions driven by solar convection (e.g., Cranmer \& van Ballegooijen 2005). Some of the Alfvén waves are reflected by the spatial gradient of the Alfvén speed, and interactions between oppositely directed Alfvén waves then cause the wave energy to cascade to small scales perpendicular to the magnetic field $\left(\lambda_{\perp}\right)$. However, the correlation lengths of Alfvén wave packets in the direction of the background magnetic field $\left(\lambda_{\|}\right)$remain comparatively long, and the Alfvén-wave frequencies remain $\ll \Omega_{i}$. When the wave energy cascades to perpendicular scales $\lambda_{\perp}$ comparable to the proton gyroradius $\rho_{i}$, the wave energy begins to dissipate, heating the corona. The role of turbulence is to transform the initial wave energy into a form - fluctuations with very small perpendicular scales - that can be efficiently dissipated by kinetic processes.

One prediction of these models is that the character of the turbulent fluctuations changes with decreasing $\lambda_{\perp}$. At $\lambda_{\perp} \gg d_{i}$, the fluctuations are non-compressive Alfvén waves, where $d_{i}=v_{A} / \Omega_{i}$ is the ion inertial length. ${ }^{5}$ On the other hand, in low- $\beta$ plasmas at $\rho_{i} \lesssim \lambda_{\perp} \lesssim d_{i}$, the fluctuations are highly compressive, in the sense that $\delta n / n_{0}>\delta B / B_{0}$, where $\delta n$ and $n_{0}$ are the fluctuating and background electron densities, and $\delta \mathbf{B}$ and $\mathbf{B}_{0}$ are the fluctuating and background magnetic fields. For $\beta \sim 1, \delta n / n_{0} \sim \delta B / B_{0}$ at $\lambda_{\perp} \sim \rho_{i}$. At even smaller scales, $\lambda_{\perp} \lesssim \rho_{i}$, the ion and electron velocities decouple for all $\beta$, the waves become dispersive, and the fluctuations produce parallel electric and magnetic field perturbations that cause the waves to damp. At $\lambda_{\perp} \lesssim \rho_{i}$, these solutions to the

\footnotetext{
${ }^{5}$ For reference, $d_{i} \simeq \beta^{-1 / 2} \rho_{i}, \beta=8 \pi p / B^{2} \ll 1$ in the corona, and for typical coronal-hole parameters $d_{i} \sim 3 \times 10^{5} \mathrm{~cm}$ at $r \simeq 5 R_{\odot}$; by contrast, $\beta \sim 0.2-1$ in the near-Earth solar wind at $\sim 1 \mathrm{AU}$ and $d_{i} \sim 3 \times 10^{6} \mathrm{~cm}$.
} 
Alfvén-wave branch of the linear dispersion relation are called kinetic Alfvén waves (KAWs). Many of the measured properties of the magnetic and electric field fluctuations in the nearEarth solar wind are consistent with KAW turbulence on small scales (e.g., Howes et al. 2008a, 2008b; Sahraoui et al. 2009, and references therein).

A very important, but somewhat indirect, test of the lowfrequency turbulence model is provided by measurements of ion and electron temperatures, both in situ and in coronal holes (the open-field-line regions from which the fast solar wind is thought to emanate). Observations with UVCS show that $T_{\perp} \gg T_{\|}$for minor ions such as $\mathrm{O}^{+5}$ in coronal holes, where $T_{\perp}$ and $T_{\|}$are the temperatures corresponding to thermal motions perpendicular and parallel to the background magnetic field $\mathbf{B}_{0}$ (Kohl et al 1998, Antonucci et al 2000). It has not yet been possible to unequivocally determine whether protons have the same temperature anisotropy in the corona. However, in situ measurements of the fast solar wind show that $T_{\perp}>T_{\|}$for the core of the proton distribution function, although the anisotropy is less pronounced for larger $\beta$ (Marsch, Ao, \& Tu 2004; Hellinger et al. 2006).

It is not yet clear whether low-frequency Alfvénic turbulence can explain perpendicular ion heating and the preferential heating of minor ions. Dissipation of Alfvénic turbulence occurs at small scales, at which the amplitude of the fluctuations is very small. The hot-plasma dispersion relation for small-amplitude waves thus provides a plausible first estimate for how KAW turbulence dissipates in the collisionless corona and solar wind. This predicts negligible damping for oblique Alfvén waves with $k_{\perp} \rho_{i} \ll 1$, where $k_{\perp}$ and $k_{\|}$are the wavevector components perpendicular and parallel to $\mathbf{B}_{0}$ (Barnes 1966). As a result, the energy cascades to scales $\lesssim \rho_{i}$. The damping of the resulting KAWs with $k_{\perp} \gg\left|k_{\|}\right|$and frequencies $\ll \Omega_{i}$ arises from Landau and/or transit-time damping and leads to parallel electron heating for $\beta \lesssim 1$, not perpendicular ion heating (Quataert 1998; Quataert \& Gruzinov 1999; Leamon et al 1999; Cranmer \& van Ballegooijen 2003; Gary \& Nishimura 2004; Howes et al 2008a). Nevertheless, a number of mechanisms have been proposed that might produce perpendicular ion heating from low-frequency turbulence, including heating by reconnection electric fields (Dmitruk, Matthaeus, \& Seenu 2004; see, however, Lehe, Parrish, \& Quataert 2009), secondary plasma instabilities triggered by the KAW velocity shear (Markovskii et al 2006), ion heating by electron phase-space holes generated by the heating of electrons (Matthaeus et al 2003; Cranmer \& van Ballegooijen 2003), and perpendicular ion heating through stochastic ion orbits (Johnson \& Cheng 2001). An outstanding problem is whether any of these mechanisms (or others) can quantitatively account for the measured perpendicular ion heating in the context of low-frequency turbulence models - this is a particularly important question given the growing body of evidence that the magnetic and electric field fluctuations at $\sim 1$ AU are consistent with low-frequency anisotropic Alfvén waves and KAWs (e.g., Sahraoui et al. 2009).

The goal of this paper is to quantitatively assess a second test of the importance of low-frequency turbulence in the solar wind: the measured power spectrum of density fluctuations. Coles \& Harmon (1989) measured the spectrum of electron density fluctuations in the corona at radii as small as $5 R_{\odot}$ using Venus as a background radio source. In addition, measurements of density fluctuations in the solar wind at $\sim 1 \mathrm{AU}$ have been carried out by a variety of methods (e.g., Celnikier et al. 1983, 1987; Hnat et al. 2005; Kellogg \& Horbury 2005). Because oblique Alfvén waves become increasingly compressive for $k_{\perp} d_{i} \sim 1$, the observed density fluctuation spectrum constrains the spectrum of oblique Alfvén waves and provides an upper limit on the heating rate $\varepsilon$ from Alfvénic turbulence. In $\S 2$, we summarize the predicted density fluctuations induced by low-frequency Alfvénic turbulence, and describe some of the remaining uncertainties in these predictions (see also Schekochihin et al. 2009). We then show how the measured density fluctuations in coronal holes and at $\sim 1$ AU constrain the heating rate $\varepsilon$ due to Alfvénic turbulence $(\$ 3$ ). In $\$ 4$ we describe the "sweeping" model for coronal heating (e.g., Schwartz et al 1981; Axford \& McKenzie 1992; Marsch \& Tu 1997; Ruzmaikin \& Berger 1998), in which the corona is heated by cyclotron damping of $\mathrm{kHz}$ waves launched directly from the Sun; we present a simple explanation for why radio observations do not rule out turbulent heating models, even though they appear to rule out the sweeping model (as was shown by Hollweg 2000). In $\S 5$ we summarize and discuss our results. Our analysis and results are broadly similar to those of Harmon \& Coles (2005), but we consider a different model for the turbulent cascade of Alfvén waves, and apply the density fluctuation constraint both to remote observations of turbulence in coronal holes and to in situ measurements of turbulence in the near-Earth solar wind.

\section{PREDICTED DENSITY FLUCTUATIONS IN LOW-FREQUENCY ALFVÉNIC TURBULENCE MODELS}

In this section we briefly summarize the density fluctuations produced by low-frequency Alfvénic turbulence (see Lithwick \& Goldreich 2001 and Schekochihin et al. 2009 for a more comprehensive discussion). We assume that the Alfvén wave power spectrum follows the "critical balance" theory of Goldreich \& Sridhar (1995). Initially we also assume that the turbulence is "balanced," i.e., that there are equal fluxes of Alfvén waves propagating towards and away from the Sun in the plasma frame, or equivalently, that there is zero cross helicity; we discuss the effects of a non-zero cross helicity at the end of the section.

Although Alfvén waves themselves are not compressive when $k_{\perp} d_{i} \gg 1$, low-frequency Alfvénic turbulence nonetheless produces significant density fluctuations, via two different physical processes. First, as energy cascades to scales $\lesssim d_{i}$, Alfvén waves transition to KAWs, which are compressive (see Fig. 3 discussed below). Second, both slow waves and entropy modes are passively mixed by the Alfvénic cascade (Lithwick \& Goldreich 2001). Because the Alfvénic fluctuations have an anisotropic Kolmogorov spectrum, the density fluctuations associated with the entropy modes and/or slow waves also have an anisotropic Kolmogorov spectrum.

For the collisionless conditions appropriate to the solar corona and solar wind, the entropy modes and slow waves are both damped, but their damping rates are $\sim k_{\|} v_{t h, p}$, where $v_{t h, p}$ is the proton thermal speed. ${ }^{6}$ The cascade rate is $\sim$ $k_{\perp} \delta v_{k} \sim v_{\mathrm{A}} k_{\|}$, where $\delta v_{k}$ is the rms amplitude of the velocity fluctuation at a perpendicular scale $k^{-1}$ (where $k \simeq k_{\perp}$ since $\left.k_{\perp} \gg k_{\|}\right)$. The cascade rate is thus comparable to the linear Alfvén wave frequency. For $\beta \lesssim 1$, the slow waves and entropy modes cascade faster than they are damped, and the

\footnotetext{
${ }^{6}$ Under these conditions, there are really two entropy modes, one associated with the electrons and one associated with the protons. The former is strongly damped, at a rate $\sim k v_{t h, e}$, while the latter is less strongly damped. We focus on the latter throughout.
} 


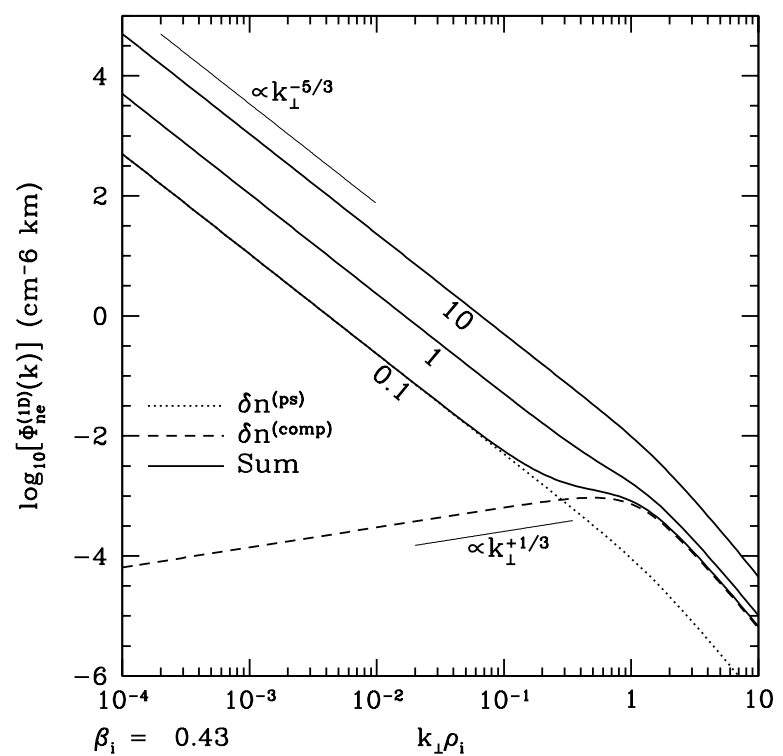

FIG. 1.- One-dimensional density fluctuation power spectrum $\Phi_{\text {ne }}^{(1 D)}$ produced by balanced low-frequency Alfvénic turbulence in the near-Earth solar wind with $\beta_{i}=0.43$ and $T_{i} / T_{e}=1$. Total density fluctuation spectra (solid) are shown for $f=0.1,1,10$, with the separate passive-scalar component (dotted) and "active" KAW component (dashed) shown explicitly for $f=0.1$.

passive scalar contribution to the density fluctuations extends to small scales $\sim \rho_{i}$ (Lithwick \& Goldreich 2001). We discuss the case of $\beta \gtrsim 1$ below.

Figure 1 summarizes our prediction for the 1-D density fluctuation spectrum $\Phi_{n e}^{(1 D)}$ associated with balanced (zero cross-helicity) low-frequency Alfvénic turbulence. The rms density fluctuations associated with the slow waves and entropy modes are cascaded by the Alfvénic fluctuations like a passive scalar and, at perpendicular scale $k^{-1}$, are denoted by $\delta n_{k}^{(\mathrm{ps})}$; the rms density fluctuations that actively arise from the kinetic Alfvén wave compressions are denoted by $\delta n_{k}^{\text {(comp) }}$. At large scales, the passive-scalar density fluctuation spectrum is chosen to be proportional to the perpendicular kinetic energy spectrum ${ }^{7}$ associated with the perpendicular velocity fluctuation $\delta v_{\perp k}$. The latter is determined using the analytic cascade model of Howes et al. (2008a), which is based on the assumptions of local nonlinear energy transfer in wavenumber space together with the critical balance between linear propagation and nonlinear interaction times. The analytic model smoothly transitions from anisotropic Kolmogorov Alfvénwave turbulence at $k_{\perp} \rho_{i} \ll 1$ to anisotropic KAW turbulence at $k_{\perp} \rho_{i} \gg 1$; it agrees well with numerical simulations of kinetic turbulence in the (limited) comparisons available to date (Howes et al. 2008b).

The relative magnitude of the passive and active density fluctuations is uncertain and may vary with position (and time) in the solar wind. We determine the constant of proportionality between the passive-scalar density spectrum and

\footnotetext{
${ }^{7}$ For small scales $k_{\perp} \rho_{i} \gtrsim 1$, the passive-scalar density spectrum follows perpendicular magnetic energy spectrum rather than the perpendicular kinetic energy spectrum.
}

the kinetic energy spectrum by specifying the ratio

$$
f \equiv\left[\frac{\delta n_{k}^{(\mathrm{ps})} / n_{0}}{\delta v_{\perp k} / v_{A}}\right]_{k=k_{0}}^{2},
$$

where $2 \pi / k_{0}$ is the driving scale or outer scale of the turbulence. Figure 1 presents 1-D density spectra from solutions of the cascade model for near-Earth solar wind conditions. Plasma parameters for this figure have been chosen to correspond to period II of Celnikier et al. (1987): $B_{0}=1.5 \times 10^{-4} \mathrm{G}, n_{i}=18 \mathrm{~cm}^{-3}, T_{i}=T_{e}=1.45 \times 10^{5} \mathrm{~K}$, and $v_{s w}=460 \mathrm{~km} / \mathrm{s}$, giving $\rho_{i}=2 \times 10^{6} \mathrm{~cm}$. We assume a driving scale $2 \pi / k_{0}=2 \times 10^{11} \mathrm{~cm}$, giving cascade model parameters $\beta_{i}=0.43, T_{i} / T_{e}=1$, and $k_{0} \rho_{i}=6 \times 10^{-5}$.

Figure 1 shows the total density fluctuation spectra (solid) for $f=0.1,1,10$, with the separate passive-scalar component (dotted) and "active" KAW component (dashed) shown explicitly for the $f=0.1$ case. The $f=0.1$ case demonstrates that the passively mixed density fluctuations have a Kolmogorov power-law spectrum at large scales (small $k$ ) with a break at $k_{\perp} \rho_{i} \sim 1$ where the turbulence transitions to dispersive KAWs. On small scales, however, the "active" density fluctuations due to KAWs become important and can dominate over the passive contribution. The density fluctuation spectrum associated with the KAWs is a factor of $\sim k_{\perp}^{2}$ flatter than Kolmogorov for $k_{\perp} \rho_{i} \lesssim 1$. Indeed, the 1D density-fluctuation spectrum from KAW compressions is rising, $\propto k_{\perp}^{1 / 3}$, for $k_{\perp} \rho_{i} \lesssim 1$. This is because the density perturbation due to a linear KAW is $\propto k_{\perp}$ times the velocity perturbation in this limit (see eq. [2] below).

The $f=0.1$ result in Figure 1 may be compared directly 8 to the measured density spectrum for this period of solar wind turbulence shown in Figure 5 of Celnikier et al. (1987). The predicted density spectrum, combining both a passive-scalar contribution and compressive KAWs, qualitatively reproduces the measured spectrum. Note that the assumption of balanced turbulence (zero cross helicity) is not an unreasonable assumption for this interval of relatively slow and dense solar wind. In addition, the value $f=0.1$ is consistent with several observations that are discussed following equation (3), below.

In coronal holes, where $\beta_{i} \ll 1$, the compressive motions associated with the KAWs become comparatively stronger. Figure 2 presents cascade-model results analogous to Figure 1 for parameters appropriate to coronal holes: $\beta_{i}=0.01$, $T_{i} / T_{e}=2$, and $k_{0} \rho_{i}=10^{-8}$. 9 The absolute normalization of the density fluctuations was chosen to roughly match the observations of Coles and Harmon (1989) in coronal holes. Total density fluctuation spectra (solid) are shown for $f=0.1,1,10$, with the decomposition into passive-scalar component (dotted) and "active" KAW component (dashed) included for each case. The results in Figure 2 are similar to those in Figure 1 except that the active KAW component of the density fluctuations is more prominent at low $\beta_{i}$. The questionable assumption of balanced (zero cross-helicity) turbulence in coronal holes is discussed in detail at the end of this section.

Figure 3 focuses on the density fluctuations due to linear KAWs, showing results as a function $k_{\perp} \rho_{i}$ for several $\beta_{i}$. For

\footnotetext{
8 Note that a value of $k_{\perp} \rho_{i}=1$ in Figure 1 corresponds to a frequency of 3.6 Hz in Figure 5 of Celnikier et al. (1987).

${ }^{9}$ In the cascade model, $k_{0}$ is not the actual outer-scale wavenumber. Instead, in the case of the corona, it is the much smaller wavenumber at which the anisotropic power spectrum would extrapolate to isotropic fluctuations with $\delta B=B_{0}$ (Howes et al 2008a).
} 


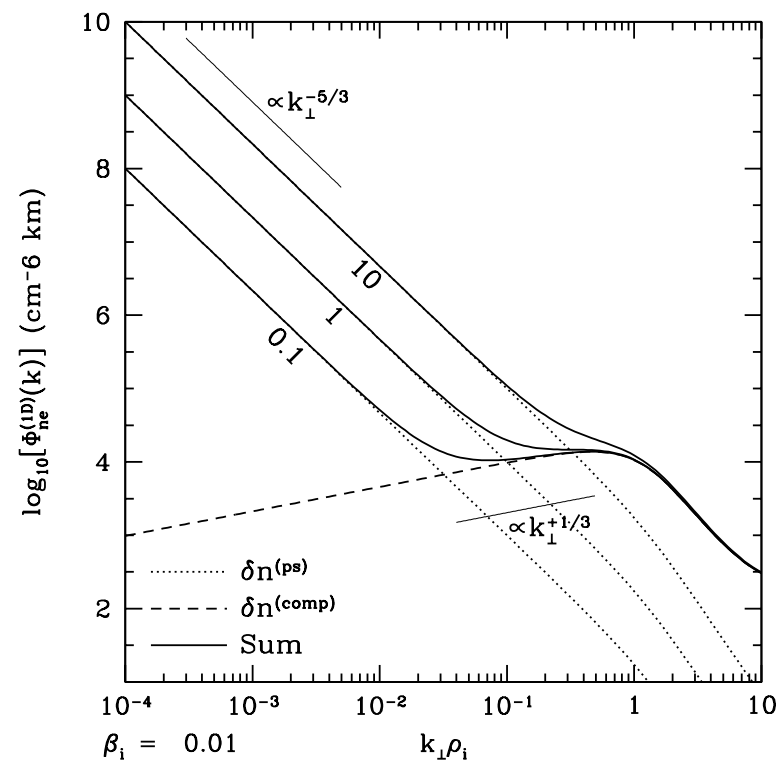

FIG. 2.- One-dimensional density fluctuation power spectrum $\Phi_{\text {ne }}^{(1 D)}$ produced by low-frequency Alfvénic turbulence in coronal holes with $\beta_{i}=0.01$ and $T_{i} / T_{e}=2$. Total density fluctuation spectra (solid) are shown for $f=$ $0.1,1,10$, with the separate passive-scalar component (dotted) and "active" KAW component (dashed) shown explicitly for the each case.

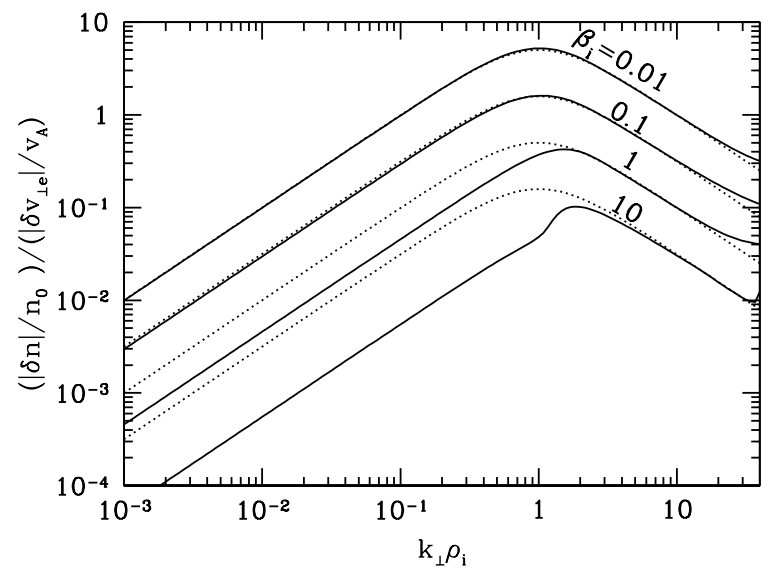

FIG. 3.-Density fluctuations relative to the perpendicular electron velocity fluctuations $\left(\delta n_{k} / n_{0}\right) /\left(\delta v_{\perp k e} / v_{A}\right)$ for $\beta_{i}=0.01,0.1,1,10$ (solid) based on linear Vlasov-Maxwell theory for kinetic Alfvén waves. Dotted lines are the predictions given by equation [2] with $\gamma_{i}=1$.

$\beta \lesssim 1$, the relation between the density and velocity fluctuations of a KAW at wavevector $\mathbf{k}$, denoted $\delta n_{k}$ and $\delta v_{\perp k}$, can be derived analytically and is given by (Hollweg 1999, Eqn. 51)

$$
\left|\frac{\delta n_{k}}{n_{0}}\right|=\frac{k_{\perp} d_{i}}{\left(1+\gamma_{i} k_{\perp}^{2} \rho_{i}^{2}\right)}\left|\frac{\delta v_{\perp k}}{v_{A}}\right|,
$$

assuming that $k_{\perp} \gg\left|k_{\|}\right|$and $\omega \ll \Omega_{i}$, where $\omega$ is the wave frequency and $\gamma_{i}$ is the adiabatic index of the ions. In addition to showing the (normalized) ratio of density fluctuations to velocity fluctuations from linear kinetic theory (solid), Figure 3 also shows the values predicted by equation (2) (dotted) with $\gamma_{i}=1$. This figure demonstrates that the density fluctuations are suppressed by a factor of $\sim \beta$ when $\beta \gtrsim 1$, as expected because the fluctuations become increasingly incompressible for high $\beta$. An additional change in the predicted density fluctuations for $\beta \gtrsim 1-$ as can occur in the solar wind at $\sim 1 \mathrm{AU}-$ is that the damping time of slow waves and entropy modes becomes shorter than their expected cascade times. Because the solar wind is collisionless, this occurs even on large scales. As a result, the passive contribution to the density fluctuations may be significantly diminished when $\beta \gtrsim 1$ (Lithwick \& Goldreich 2001).

Equation (2) can be used to estimate the ratio between the passive-scalar density fluctuation and the density fluctuation arising from KAW compressions. We assume that $\delta n_{k}^{\text {(comp) }} / \delta v_{\perp k}$ equals the ratio $\delta n_{k} / \delta v_{\perp k}$ for linear KAWs given by equation (2). When $\beta \lesssim 1$ and the cross helicity is zero, $\delta n_{k}^{(\mathrm{ps})} / \delta v_{\perp k}$ is independent of $k$ within the inertial range. Assuming that $\delta n_{k}^{(\mathrm{ps})} / \delta v_{\perp k}$ remains roughly constant from the outer scale all the way to $k_{\perp} \sim \rho_{i}^{-1}$, equation (2) implies that for balanced turbulence with $\beta \lesssim 1$

$$
\left[\frac{\delta n_{k}^{(\mathrm{comp})}}{\delta n_{k}^{(\mathrm{ps})}}\right]_{k=\rho_{i}^{-1}} \sim(\beta f)^{-1 / 2},
$$

At $1 \mathrm{AU}$, the value of $\delta n_{k}^{(\mathrm{ps})} / n_{0}$ at $k=k_{0}$ ranges from roughly 0.1 to 0.3 , as the assumed outer-scale time scale (as measured in the spacecraft frame) of the turbulence is varied from $1-12$ hrs (Tu \& Marsch 1995, Fig. 2-14). If we set $v_{\mathrm{A}}=77 \mathrm{~km} / \mathrm{s}$ as in Celnikier's period II data and $\delta v_{\perp k} \simeq 30 \mathrm{~km} / \mathrm{s}$ at $k=k_{0}$ at $1 \mathrm{AU}$ (see Cranmer \& van Ballegooijen 2005, Fig.9), then $f \simeq 0.07-0.6$. Equation (3) thus suggests that KAW compressions usually dominate the density fluctuations on small scales $\left(k_{\perp} \rho_{i} \sim 1\right)$ at $1 \mathrm{AU}$.

It is important to note several theoretical uncertainties in our predictions for the density fluctuations that should accompany low frequency Alfvénic turbulence in the solar wind. First, the relative amplitudes of the slow waves, entropy modes, and Alfvén waves on large scales are not precisely known. Therefore, we present several values of $f$ in Figures 11 and 2 , A second, and more significant, limitation is that we have assumed that the turbulence is "balanced" (zero cross-helicity), i.e., that the energy in waves propagating towards the sun (in the solar-wind frame) equals the energy in waves propagating away from the sun. This is generally not observed to be true at $\sim 1$ AU (particularly in the fast wind; Grappin et al. 1990; Tu \& Marsch 1990). Closer to the Sun there is an even larger difference between the energies of Sunward and anti-Sunward waves (Roberts et al 1987; Bavassano et al 2000). Non-zero cross helicity or "imbalance" affects strong Alfvénic turbulence in several ways. For example, nonlinear interactions among Alfvén waves occur only between waves propagating in opposite directions in the plasma frame (Iroshnikov 1963, Kraichnan 1965), and so if the energy in waves propagating towards the Sun is very small, then the energy cascade rate and turbulent heating rate also become small (Dobrowolny et al 1980, Hossain et al 1995, Chandran et al 2009). Finite cross helicity may also modify the wavenumber scalings of the inertial-range power spectra of the density, magnetic field, and velocity. Because the "minority" Sunward Alfvén waves and the passive scalar fluctuations are both cascaded by the "dominant" anti-Sunward Alfvén waves, the passive scalar spectrum is expected to have the same inertial-range scaling as the Sunward waves (Lithwick \& Goldreich 2003; Chandran 2008b). In some studies of Alfvénic turbulence with cross helicity (e.g., Grappin et al 1983; Chandran 2008a; 
Beresnyak \& Lazarian 2009) the minority Alfvén waves have a shallower power spectrum than the dominant Alfvén waves, suggesting that the passive scalar spectrum is shallower than the magnetic spectrum in highly imbalanced turbulence. This finding may be related to the shallow density spectra seen in radio observations of the corona, in which the turbulence is expected to be highly "imbalanced" (Cranmer \& van Ballegooijen 2005, Verdini \& Velli 2007). ${ }^{10}$ However, a number of other studies find that the inertial-range spectra of the minority waves and dominant waves scale with wavenumber in the same way (Lithwick, Goldreich, \& Sridhar 2007; Perez \& Boldyrev 2009; Podesta \& Bhattacharjee 2009). Moreover, the different studies cited above disagree over whether the spectra of the minority and dominant waves are equal at the dissipation scale ("pinning"). Because imbalanced Alfvénic turbulence is still not fully understood, and is likely the norm in the solar wind, we are only able to derive upper limits on the turbulent heating rates from the density observations, as discussed further below. In addition, this uncertainly implies that the precise power-law scalings for $\Phi_{\mathrm{ne}}^{1 \mathrm{D}}$ in Figures 1 and 2 should not be taken too literally, although we believe that our conclusions about the relative contribution of the active and passive density fluctuations are robust.

\section{OBSERVATIONAL CONSTRAINTS ON LOW-FREQUENCY ALFVÉNIC TURBULENCE MODELS}

In this section, we discuss observational constraints on density fluctuations in the solar corona and solar wind, and their implications for low-frequency Alfvénic turbulence models.

\subsection{Coronal Holes}

Coles \& Harmon (1989) analyzed the spectral broadening of Arecibo radar observations of Venus near superior conjunction to determine the three-dimensional power spectrum of electron density fluctuations $\Phi_{n e}(r, k)$ (treated as an isotropic function of wave vector $\mathbf{k}$ ) at a range of heliocentric distances $r$ in the slow solar wind. They were able to fit $\Phi_{n e}(r, k)$ at $r=5 R_{\odot}$ with one power law at $k<10^{-7} \mathrm{~cm}^{-1}$, a slightly shallower power law at $10^{-7} \mathrm{~cm}^{-1}<k<k_{i}$, and an exponential or Gaussian at $k>k_{i}$, where

$$
k_{i} \simeq 10^{-5} \mathrm{~cm}^{-1}
$$

is the "inner-scale" wave number at $r=5 R_{\odot}$. They found that at $k=k_{i}$ and $r=5 R_{\odot}, \Phi_{n e} \simeq 9.0 \times 10^{3} \mathrm{~cm}^{-6} \mathrm{~km}^{3}$ (see their Fig. 4). We focus on the inner scale for reasons that will become clearer below. Coles et al (1991) found that $\Phi_{n e}$ is a factor of $\simeq 15$ smaller in coronal holes than in the slow wind, and thus we set

$$
\Phi_{n e}\left(r=5 R_{\odot}, k=10^{-5} \mathrm{~cm}^{-1}\right) \simeq 6.0 \times 10^{2} \mathrm{~cm}^{-6} \mathrm{~km}^{3} .
$$

The rms electron density fluctuation $\delta n_{k_{i}}$ is given by $\delta n_{k_{i}}^{2} \simeq$ $4 \pi k_{i}^{3} \Phi_{n e}\left(k_{i}\right)$, which implies $\delta n_{k_{i}} \simeq 87 \mathrm{~cm}^{-3}$ at $r=5 R_{\odot}$. We estimate the coronal-hole electron density from Eqn. (4) of Feldman et al (1997), which gives $n_{e}=5.9 \times 10^{3} \mathrm{~cm}^{-3}$ at $r=5 R_{\odot}$; this is very close to the value inferred by Fisher \& Guhathakurta (1995) from observations taken with the Spar$\tan 201-01$ coronagraph. This value for the background density then gives

$$
\frac{\delta n_{k_{i}}}{n_{0}} \simeq 1.5 \times 10^{-2}
$$

\footnotetext{
${ }^{10}$ For example, Markovskii \& Hollweg (2002) fit the one-dimensional density spectrum at $r=5 R_{\odot}$ and at $k<10^{-7} \mathrm{~cm}^{-1}$ with a power law of the form $k^{-1.2}$ and a power law of the form $k^{-2 / 3}$ at $10^{-7} \mathrm{~cm}^{-1}<k<$ $10^{-5} \mathrm{~cm}^{-1}$. The density fluctuations at $k<10^{-7} \mathrm{~cm}^{-1}$ in their study are thought to correspond to inertial-range passive-scalar fluctuations.
}

at $r=5 R_{\odot}$.

An upper limit on the rms amplitude of the Alfvénic velocity fluctuation at perpendicular scale $k_{i}^{-1}$, denoted $\delta v_{k_{i}}$, can be obtained by assuming that the density fluctuations at scale $k_{i}^{-1}$ arise entirely from KAWs. Using the linear eigenfunctions of KAWs, we can write

$$
\frac{\delta v_{k_{i}}}{v_{A}} \lesssim\left(\frac{1+\gamma_{i} k_{i}^{2} \rho_{i}^{2}}{k_{i} d_{i}}\right) \frac{\delta n_{k_{i}}}{n_{0}} .
$$

If the compressibility of the KAWs noticeably affects the density spectrum from $10^{-7} \mathrm{~cm}^{-1}<k<10^{-5} \mathrm{~cm}^{-1}$, as conjectured above and as is suggested by Figure 2 , then $\delta v_{k_{i}}$ may be close to the upper limit given in equation (7); this is because the fraction of $\Phi_{n e}$ that arises from KAWs increases at larger $k$ (Fig. 2). To evaluate the right-hand side of equation (7) we assume $\gamma_{i}=1$ and $T_{i}=2.0 \times 10^{6} \mathrm{~K}$ and adopt the coronal-hole magnetic field model of Cranmer \& van Ballegooijen (2005) (their eq. 2), which gives $B_{0}=5.8 \times 10^{-2} \mathrm{G}$ at $r=5 R_{\odot}$. These parameters give $\rho_{i}=2.3 \times 10^{4} \mathrm{~cm}, d_{i}=3 \times 10^{5} \mathrm{~cm}$, $v_{\mathrm{A}}=1.6 \times 10^{8} \mathrm{~cm} / \mathrm{s}$, and

$$
\frac{\delta v_{k_{i}}}{v_{A}} \lesssim 0.35\left(\frac{\delta n_{k_{i}}}{n_{0}}\right)
$$

or, equivalently,

$$
\delta v_{k_{i}} \lesssim 8.4 \mathrm{~km} / \mathrm{s}
$$

At $k=k_{i}$, the density spectrum at $r=5 R_{\odot}$ is still close to the value obtained by extrapolating a power-law fit to $\Phi_{n e}$ for values of $k$ between $10^{-7} \mathrm{~cm}^{-1}$ and $10^{-6} \mathrm{~cm}^{-1}$. We can thus assume that most of the cascade power is still present at $k_{\perp}=k_{i}$ and that most of the dissipation occurs at $k_{\perp}>k_{i}$. Moreover, because $k_{i} \rho_{i} \simeq 0.2$, the kinetic energy and magnetic energy of Alfvénic fluctuations at $k_{\perp}=k_{i}$ are comparable, as in incompressible MHD, but not like the short-wavelength regime $k_{\perp} \rho_{i} \gg 1$, in which the magnetic energy dominates. The energy density of Alfvénic fluctuations at scale $k_{i}^{-1}$ is thus $\simeq \rho \delta v_{k_{i}}^{2}$. The time required for the fluctuation energy at $k_{\perp}=k_{i}$ to cascade to $k_{\perp} \geq 2 k_{i}$, denoted $t_{c}$, satisfies the inequality

$$
t_{\mathrm{c}} \gtrsim\left(k_{i} \delta v_{k_{i}}\right)^{-1} .
$$

The right-hand side of equation (10) is the shearing time scale for an Alfvénic velocity fluctuation at $k_{\perp}=k_{i}$ with rms amplitude $\delta v_{k_{i}}$. This is a lower-limit on $t_{\mathrm{c}}$ because in incompressible MHD, the anti-Sunward waves are sheared by waves propagating towards the Sun (Iroshnikov 1963; Kraichnan 1965), as summarized in $\$ 2$. Thus, if the Sunward waves are much less energetic than the anti-Sunward waves at $k_{\perp}=k_{i}$, the time required for Sunward waves to shear and substantially distort anti-Sunward waves is much greater than $\left(k_{i} \delta v_{k_{i}}\right)^{-1}$ (Lithwick, Goldreich, \& Sridhar 2007; Beresnyak \& Lazarian 2008; Chandran 2008a). The cascade power in lowfrequency Alfvén-wave turbulence at $r=5 R_{\odot}$, denoted $\varepsilon$ is roughly $\rho v_{k i}^{2} / t_{\mathrm{c}}$. Given equation (10), we can write

$$
\varepsilon \leq c_{0} \rho k_{i} \delta v_{k_{i}}^{3},
$$

where $c_{0} \simeq 0.25$ in strong incompressible MHD turbulence with equal fluxes of Alfvén waves propagating parallel and anti-parallel to the background magnetic field (Howes et al. 2008a). Substituting equation (9) into equation (11), we find

$$
\varepsilon \lesssim 1.5 \times 10^{-8} \mathrm{erg} \mathrm{cm}^{-3} \mathrm{~s}^{-1} .
$$


This upper limit can be compared with the parameterized heating rates employed in empirical models of the fast solar wind. Allen et al (1998) constructed a series of twofluid models with heating rates chosen to match in situ measurements of the fast wind at $1 \mathrm{AU}$ as well as the coronal-hole density profile inferred from the brightness profile of electron-scattered, polarized white light (Fisher \& Guhathakurta 1995). In these models, denoted SW2, SW3, and SW4, the total (electron plus proton) heating rates at $r=$ $5 R_{\odot}$ were $3.1 \times 10^{-9} \mathrm{erg} \mathrm{cm}^{-3} \mathrm{~s}^{-1}, 1.4 \times 10^{-8} \mathrm{erg} \mathrm{cm}^{-3} \mathrm{~s}^{-1}$, and $6.8 \times 10^{-9} \mathrm{erg} \mathrm{cm}^{-3} \mathrm{~s}^{-1}$, respectively. Esser et al (1997) constructed similar models with higher temperatures at the coronal base and lower heating rates. The value of $\varepsilon$ at $r=5 R_{\odot}$ was $2 \times 10^{-10} \mathrm{erg} \mathrm{cm}^{-3} \mathrm{~s}^{-1}$ in their model $\mathrm{A}$ and $8 \times 10^{-10} \mathrm{erg} \mathrm{cm}^{-3} \mathrm{~s}^{-1}$ in their model B. ${ }^{11}$ Since these models (by construction) provide a reasonable fit to the observed properties of the fast wind, we take the actual heating rate in coronal holes at $r=5 R_{\odot}$ to be in the range spanned by these models, i.e., between $2 \times 10^{-10} \mathrm{erg} \mathrm{cm}^{-3} \mathrm{~s}^{-1}$ and $1.4 \times$ $10^{-8} \mathrm{erg} \mathrm{cm}^{-3} \mathrm{~s}^{-1}$. Since the upper limit in equation (12) is above this range, we conclude that a model in which the fast wind is accelerated by heating from low-frequency Alfvénic turbulence is consistent with the radio observations. Future investigations could provide tighter constraints on the fraction of $\delta n_{k_{i}}$ that arises directly from KAWs as well as the ratio of Sunward to anti-Sunward wave energy at $k_{\perp}=k_{i}$, which could in principle lower the upper limit on $\varepsilon$ that is implied by the density fluctuation measurements.

\subsection{The Solar Wind at $\sim 1 A U$}

Measurements of density fluctuations in the solar wind at $\sim 1$ AU have been carried out by a variety of methods (e.g., Celnikier et al. 1983, 1987; Hnat et al. 2005; Kellogg \& Horbury 2005); these allow us to calculate an upper limit on the heating by low-frequency Alfvénic turbulence analogous to that derived in the previous section.

For concreteness, we use Celnikier et al. (1987)'s period II to find an upper limit to the KAW contribution to the density fluctuations, but similar results are obtained from other measurements. From their Figure 7 , we infer that $\delta n_{k} / n_{0} \lesssim 10^{-2}$ at $k_{\perp} \rho_{i} \simeq 0.3,{ }^{12}$ where $B \simeq 1.5 \times 10^{-4} \mathrm{G}, n_{0} \simeq 18 \mathrm{~cm}^{-3}, T_{p} \simeq$ $1.5 \times 10^{5} \mathrm{~K}, \rho_{p} \simeq 2 \times 10^{6} \mathrm{~cm}, v_{A} \simeq 77 \mathrm{~km} \mathrm{~s}^{-1}, v_{\text {wind }} \simeq 460 \mathrm{~km}$ $\mathrm{s}^{-1}$, and $\beta \simeq 0.43$ in this epoch of data. Unlike in the observations of the solar corona described in $\$ 3.1$ there is no clear evidence for an inner scale to the density fluctuations at $1 \mathrm{AU}$. Given the lack of a preferred scale, we evaluate the density fluctuations at $k_{\perp} \rho_{i} \simeq 0.3$ as a compromise between the scales where the KAW density fluctuations peak $\left(k_{\perp} \rho_{i} \sim 1\right)$ and the scales where incompressible MHD is a reasonable model for the cascade $\left(k_{\perp} \rho_{i} \ll 1\right)$; our conclusions are, however, insensitive to reasonable variations about this choice.

A limit on the density fluctuations due to KAWs of $\delta n_{k} / n_{0} \lesssim 10^{-2}$ at $k_{\perp} \rho_{i} \simeq 0.3$ corresponds to a limit on the velocity fluctuations of $\delta v_{k} / v_{A} \lesssim 10^{-2}$ at the same scale (i.e., $\delta v_{k} \lesssim 1 \mathrm{~km} \mathrm{~s}^{-1}$ ) and thus to a limit on the heating rate due to low-frequency Alfvénic turbulence of

$$
\varepsilon \lesssim 5 \times 10^{-16} \mathrm{erg} \mathrm{cm}^{-3} \mathrm{~s}^{-1}
$$

${ }^{11}$ For comparison, the heating rate at $r=5 R_{\odot}$ was $3 \times 10^{-10} \mathrm{erg} \mathrm{cm}^{-3} \mathrm{~s}^{-1}$ in the theoretical model of Markovskii \& Hollweg (2002), and between $8 \times$ $10^{-10} \mathrm{erg} \mathrm{cm}^{-3} \mathrm{~s}^{-1}$ and $5 \times 10^{-9} \mathrm{erg} \mathrm{cm}^{-3} \mathrm{~s}^{-1}$ in the "sweeping" models of Marsch \& Tu (1997).

12 This corresponds to a satellite-frame frequency $\simeq 1 \mathrm{~Hz}$.
At $\sim 1 \mathrm{AU}$, the heating rate in the solar wind can be directly measured from the non-adiabatic temperature profile of the protons and electrons, using $\varepsilon \simeq \rho v_{r} T d s / d r$. For example, using the proton data from Voyager 2 (e.g., Matthaeus et al. 1999), we infer that $\varepsilon \simeq 3 \times 10^{-16} \mathrm{erg} \mathrm{cm}^{-3} \mathrm{~s}^{-1}$ is required to explain the non-adiabaticity of the solar wind at 1 AU. ${ }^{13}$ The close correspondence between this measured heating rate at $1 \mathrm{AU}$ and the upper limit in equation (13) implies that, if low frequency Alfvénic turbulence contributes to heating the solar wind at $\sim 1 \mathrm{AU}$, direct density fluctuations due to compressive KAWs must contribute significantly to the measured density fluctuations at $k_{\perp} \rho_{i} \sim 1$. In this context, we note that Kellogg and Horbury (2005) have argued for an ion-acoustic or KAW origin for the small-scale density fluctuations in the solar wind at $1 \mathrm{AU}$ using completely independent arguments. It is also important to note that the Celnikier et al. measurements are in the relatively slow solar wind $(v \simeq 450 \mathrm{~km}$ $\mathrm{s}^{-1}$ ), in which the turbulence is observed to be fairly balanced (Grappin et al. 1990). Thus the cascade time is likely comparable to the lower limit in equation (10), in which case the cascade power can also be comparable to the upper limit in equation (13).

The density fluctuation measurements of Celnikier et al. (1987) show a break from a Kolmogorov spectrum at low $\mathrm{k}$ to a flatter spectrum at high $\mathrm{k}$. This is qualitatively consistent with the expectations from Figure 1] The observed break happens at a rest frame frequency of $\simeq 0.1 \mathrm{~Hz}(\mathrm{Cel}-$ nikier et al. 1987), which corresponds to $k^{-1} \simeq 7 \times 10^{7} \mathrm{~cm}$ $\gg \rho_{i}, d_{i} \sim 3 \times 10^{6} \mathrm{~cm}$ using the Taylor hypothesis. This suggests that $f \ll 1$, i.e., that the passive scalar contribution to the density fluctuations is small compared to the KAW contribution, so that the latter begins to dominate at $k_{\perp} \rho_{i} \ll 1$ (see Fig. 11. A small value of $f$ at $1 \mathrm{AU}$ is not implausible given the observations described above, following equation (3).

\section{OBSERVATIONAL CONSTRAINTS ON THE "SWEEPING" MODEL OF CYCLOTRON HEATING}

An alternative coronal heating mechanism that has been considered in some detail is ion-cyclotron heating by highfrequency (kHz-range) waves. One of the proposed sources for these $\mathrm{kHz}$-range waves is magnetic reconnection in the photosphere or chromosphere (Axford \& McKenzie 1992). Because $B_{0}$ and $\Omega_{i}$ decrease with increasing $r$, waves with $\omega<\Omega_{i}$ at the base of the corona eventually reach radii at which $\omega \simeq \Omega_{i}$, at which point the waves undergo cyclotron damping and heat the ions. If a broad frequency spectrum of waves is launched from the Sun, the waves will result in radially extended ion heating, with lower-frequency waves causing heating farther from the Sun (Schwartz et al 1981; Axford \& McKenzie 1992; Marsch \& Tu 1997; Tu \& Marsch 1997; Ruzmaikin \& Berger 1998; Czechowski et al 1998). Although cyclotron heating could in principle explain the observed temperature anisotropies of ions in the corona and fast solar wind, this "sweeping" or "direct-launching" model faces a significant difficulty. If the waves are oblique, with wave vectors that make a nonzero angle $\theta$ with respect to $\mathbf{B}_{0}$, then the waves induce density fluctuations at high frequencies. Holl-

\footnotetext{
13 Cranmer \& Van Ballegooijen 2005 and Cranmer et al (2009) find similar heating rates at 1 AU. Celnikier et al. (1987) do not report the electron temperature for their solar wind epochs, but the mean electron temperature for this solar wind speed is $\simeq 1.5 \times 10^{5} \mathrm{~K}$ (Newbury et al. 1998), which is comparable to $T_{p}$. We thus expect the electron heating rate to be at most comparable to the proton heating rate; the proton heating rate is thus a reasonable proxy for the total heating rate, at the factor of 2 level.
} 
weg (2000) investigated this effect, modeling the wave obliquity by setting $\theta=60^{\circ}$. Using the wave power spectra employed in the "sweeping" models of Marsch \& Tu (1997), he found that the waves would induce larger density fluctuations than are detected by radio observations, by a factor of $>10^{2}$ at $k=0.3 \times 10^{-5} \mathrm{~cm}^{-1}$. Tu \& Marsch $(2000,2001)$ have responded to this criticism by suggesting that transit-time damping could remove the obliquely propagating waves and thereby reduce the density fluctuations. We believe, however, that "phase mixing" by laminar (Heyvaerts \& Priest 1983) and turbulent (Chandran 2008b) density structures will transfer Alfvén-wave energy to larger $k_{\perp}$, thereby significantly limiting the amount of Alfvén/ion-cyclotron wave energy that can remain at small $\theta$ as the waves propagate from the coronal base out to $r=5 R_{\odot}$.

\subsection{Why Radio Observations Rule Out the "Sweeping" Model but not Turbulent Heating}

In the "sweeping" or "direct-launching" model, the heating rate from high-frequency waves $Q_{\mathrm{hf}}$ satisfies

$$
Q_{\mathrm{hf}} \sim \frac{\mathcal{E}_{\mathrm{hf}}}{t_{\mathrm{pr}}},
$$

where $\mathcal{E}_{\mathrm{hf}}$ is the energy density of waves with frequencies between $0.5 \Omega_{i}$ and $\Omega_{i}$ and $t_{p r}$ is the time for Alfvén waves to propagate through the distance over which the magnetic field strength decreases by a factor of 2 . Neglecting the solar-wind bulk-flow speed, and assuming $B_{0} \propto r^{-2}$, the value of $t_{\mathrm{pr}}$ is $\sim 0.4 r / v_{\mathrm{A}}$, which is $\simeq 800 \mathrm{~s}$ at $r=5 R_{\odot}$ in our model coronal hole. On the other hand, in the turbulent-heating model, the heating rate for low-frequency turbulence $Q_{\text {If }}$ satisfies

$$
Q_{\mathrm{lf}} \sim \frac{\mathcal{E}_{\mathrm{lf}}}{t_{\mathrm{c}}},
$$

where $\mathcal{E}_{\mathrm{lf}}$ is the energy density of low-frequency Alfvénic fluctuations with $0.5 k_{i}<k_{\perp}<k_{i}$, and $t_{\mathrm{c}}$ is the cascade time at $k_{\perp}=k_{i}$. Equations (9) and (10) imply $t_{c} \gtrsim 0.1 \mathrm{~s}$, so that $t_{c}$ may be as much as four orders of magnitude smaller than $t_{\mathrm{pr}}$. For a fixed heating rate and $t_{\mathrm{c}} \ll t_{\mathrm{pr}}$, the turbulentheating model requires much less fluctuation energy at small scales than the sweeping model. In addition, because the compressibility of anisotropic KAWs is comparable to that of oblique ion-cyclotron waves, the turbulent-heating model also requires much lower density fluctuations than the sweeping model for a fixed heating rate when $t_{\mathrm{c}} \ll t_{\mathrm{pr}}$.

\section{CONCLUSION}

In this paper, we have used density fluctuation measurements to test models in which the solar wind is heated by low-frequency Alfvén-wave turbulence; the turbulent fluctuations are assumed to satisfy the inequality $k_{\perp} \gg\left|k_{\|}\right|$. The density fluctuation measurements - many of which are based on scintillation at radio wavelengths - place an upper limit on the amplitude of Alfvén waves at small scales, where obliqueAlfvén-wave compressions (kinetic Alfvén waves $\equiv$ KAWs) induce density fluctuations. This upper limit in turn implies an upper limit on the turbulent heating rate. We calculate this upper limit for coronal holes at $r=5 R_{\odot}$ and in the near-Earth solar wind at $1 \mathrm{AU}$. The upper limit on the turbulent heating rate we derive (eq. [11]) can be realized only if two conditions are satisfied: (1) most of the measured small-scale density fluctuations are in fact from KAWs, and (2) the timescale for energy to cascade from one scale to another is $\simeq\left(k_{\perp} \delta v_{k_{\perp}}\right)^{-1}$.
The latter is only expected to be the case if there is comparable energy in Sunward and anti-Sunward waves in small-scale turbulent fluctuations. It is currently unclear whether this is in fact the case, both theoretically and empirically (especially at $\sim 5 R_{\odot}$ ), which is one of the primary reasons that density fluctuations can only place an upper limit on the heating rate produced by low-frequency Alfvénic turbulence (in their related analysis Coles \& Harmon 2005 implicitly assumed that the turbulence is balanced).

The upper limit on the turbulent heating rate at $r=5 R_{\odot}$ in coronal holes exceeds the parameterized turbulent heating rates employed in models of the fast solar wind, by a factor of a few to $\sim 100$ depending on the models (\$3.1). At $1 \mathrm{AU}$, on the other hand, the upper limit on the turbulent heating rate due to low-frequency Alfvénic turbulence is within a factor of 2 of the measured heating rate - the latter being from the measured non-adiabatic temperature profiles $(\$ 3.2)$. We thus conclude that models in which the solar wind is accelerated by heating from low-frequency Alfvénic turbulence are consistent with the measured density fluctuations in the solar wind. By contrast, models in which the solar wind is accelerated by non-turbulent high frequency waves "sweeping" through the ion-cyclotron resonance are inconsistent with the measured density fluctuations at $5 R_{\odot}$ unless the ion-cyclotron waves have $k_{\perp}=0$ to very high precision (Hollweg 2000). The key difference between the turbulent model and the sweeping model is that in the sweeping model the timescale to dissipate the energy contained in small-scale $\left(\sim \rho_{i}\right)$ fluctuations is set by the expansion speed of the solar wind, while in the turbulence model it is set by the much faster cascade time at small scales $(4.1)$. Thus for a fixed heating rate the turbulent heating model has a much smaller energy density in small-scale fluctuations, which in turn produce much smaller density fluctuations.

Future measurements and calculations could help to clarify the relative importance of passive fluctuations and KAW compressions in producing density fluctuations at different scales for different values of $\beta$. For example, the passive scalar contribution to the density fluctuations in the solar wind should be suppressed when $\beta \gtrsim 1$, because the damping rate of the passive fluctuations (slow waves and entropy modes) is larger than the cascade rate $(\$ 2)$. Thus, measurements of the density fluctuations in solar wind epochs with large $\beta$ are likely to be particularly instructive; more detailed theoretical calculations of the suppression of the passive scalar contribution at $\beta \gtrsim 1$ would aid in interpreting such measurements. In addition, future progress towards understanding the inertial-range power spectra of the density fluctuations, Sunward Alfvén waves, and anti-Sunward Alfvén waves in imbalanced (or cross-helical) turbulence could lead to significantly tighter constraints on the heating rate contributed by KAW turbulence, particularly near the Sun where the energy in anti-Sunward Alfvén waves greatly exceeds the energy of Sunward Alfvén waves. Finally, the fact that the upper limit on the KAW heating rate at $1 \mathrm{AU}$ derived from density fluctuations is nearly equal to the measured heating rate in the solar wind (\$3.2) strongly motivates a more detailed analysis of the small-scale density fluctuations and their implications.

This work was supported in part by the the Center for Integrated Computation and Analysis of Reconnection and Turbulence (CICART) under DOE under grant number DEFG02-07-ER46372, by the NSF/DOE Partnership in Basic 
Plasma Science and Engineering under grant number AST0613622, and by NASA under grant numbers NNX07AP65G and NNH06ZDA001N-SHP06-0071 at the University of New
Hampshire. E. Quataert was supported in part by NSF-DOE Grant PHY-0812811 and by NSF Grant ATM-0752503.

\section{REFERENCES}

Abraham-Shrauner, B. \& Feldman, W. C. 1977, J. Geophys. Res., 82, 618 Allen, L. A., Habbal, S. R., \& Hu, Y. Q. 1998, J. Geophys. Res., 103, 6551 Antonucci, E., Dodero, M. A., \& Giordano, S. 2000, Solar Phys., 197, 115 Axford, W. I. \& McKenzie, J. F. 1992, in Solar Wind Seven, ed. Marsch, E. \& Schwenn, R. R. (New York: Pergamon, p. 1)

Barnes, A. 1966, Phys. Fluids, 9, 1483

Bavassano, B., Pietropaolo, E., \& Bruno, R. 2000, J. Geophys. Res., 105, 15959

Belcher, J. W., \& Davis., L. J., \& Smith, E. J. 1969, J. Geophys. Res., 74, 2302

Beresnyak, A., \& Lazarian, A. 2008, ApJ, 682, 1070

Boldyrev, S. 2006, Phys. Rev. Lett., 96115002

Celnikier, L. M., Harvey, C. C., Jegou, R., Kemp, M., \& Moricet, P. 1983, A\& A, 126, 293

Celnikier, L. M., Muschietti, L., \& Goldman, M.V. 1987, A\& A, 181, 138

Chandran, B. D. G. 2005, Phys. Rev. Lett., 95, 265004

Chandran, B. D. G. 2008a, ApJ, 685, 646

Chandran, B. D. G. 2008b, Phys. Rev. Lett., 101, 235004

Chandran, B. D. G., Quataert, E., Howes, G. G., Hollweg, J. V., and Dorland, W. 2009, ApJ, in press.

Coleman, P. J. 1968, ApJ, 153, 371

Coles, W. A., \& Harmon, J. K. 1989, ApJ, 337, 1023

Coles, W. A., Grall, R. R., \& Klinglesmith, M. T. 1995, J. Geophys. Res. 100,17069

Cranmer, S. R. 2000, ApJ, 532, 1197

Cranmer, S. R. \& van Ballegooijen, A. A. 2003, ApJ, 594, 573

Cranmer, S. R. \& van Ballegooijen, A. A. 2005, ApJS, 156, 265

Cranmer, S. R., van Ballegooijen, A. A., \& Edgar, R. J. 2007, ApJS, 171, 520

Cranmer, S. R., Matthaeus, W. H., Breech, B., \& Kasper, J. C. 2009, ApJ, in press

Czechowski, A., Ratkiewicz, R., McKenzie, J. F., \& Axford, W. I. 1998, A\& A, 335, 303

Dmitruk, P., Milano, L. J., \& Matthaeus, W. H. 2001, ApJ, 548, 482

Dmitruk, P., Matthaeus, W. H., Milano, L. J., Oughton, S., Zank, G. P., \& Mullan, D. J. 2002, ApJ, 575, 571

Dmitruk, P., Matthaeus, W. H., \& Seenu, N. 2004, ApJ, 617, 667

Dobrowolny, M., Mangeney, A., Veltri, P. L. 1980, Phys. Rev. Lett., 76, 3534

Feldman, W. C., Habbal, S. R., Hoogeveen, G., \& Wang, Y.-M. 1997, J.

Geophys. Res., 102, 26905

Fisher, R. \& Guhathakurta, M. 1995, ApJL, 447, 139

Fisk, L. A. 2003, J. Geophys. Res., 108, 7

Fisk, L. A., Gloeckler, G., Zurbuchen, T. H., Geiss, J., \& Schwadron, N. A. 2003, Solar Wind Ten, Am. Inst. Phys. Conf. Ser., 679, eds. Velli, M., Bruno, R., Malara, F., \& Bucci, B., 287

Gary, S. P. \& Nishimura, K. 2004, J. Geophys. Res., 109, 2109

Goldreich, P., \& Sridhar, S. 1995, ApJ, 438, 763

Grappin, R., Pouquet, A., \& Léorat, J. 1983, A\&A, 126, 51

Grappin, R., Mangeney, A., \& Marsch, E. 1990, J. Geophys. Res., 958197

Gruzinov, A. 1998, ApJ, 501, 787

Harmon, J., \& Coles, W. 2005, J. Geophys. Res., 110, A03101

Hartle, R. E., \& Sturrock, P. A. 1968, ApJ, 151, 1155

Hellinger, P., Trávníček, P., Kasper, J. C., \& Lazarus, A. J. 2006, Geophys. Res. Lett., 33, 9101

Heyvaerts, J. \& Priest, E. R. 1983, A\& A, 117, 220

Hollweg, J. V. 1999, J. Geophys. Res., 104, 14811

Hollweg, J. V. 2000, J. Geophys. Res., 105, 7573

Hollweg, J. V., \& Isenberg, P. A. 2002, J. Geophys. Res., 107, 1

Hollweg, J. V. \& Turner, J. M. 1978, J. Geophys. Res., 83, 97

Holzer, T. 1977, J. Geophys. Res., 82, 23

Hossain, M., Gray, P. C., Pontius, D. H., Matthaeus, W. H., \& Oughton, S. 1995, Phys. Fluids, 7, 2886

Howes, G. G., Cowley, S. C., Dorland, W., Hammett, G. W., Quataert, E., \& Schekochihin, A. A. 2008a, J. Geophys. Res., 113, A05103

Howes, G. G., Dorland, W., Cowley, S. C., Hammett, G. W., Quataert, E., Schekochihin, A. A., \& Tatsuno, T. 2008b, Phys. Rev. Let., 100, 65004
Iroshnikov, P. 1963, Astron. Zh. 40, 742

Isenberg, P. A. \& Hollweg, J. V. 1983, J. Geophys. Res., 88, 3923

Johnson, J. R. \& Cheng, C. Z. 2001, Geophys. Res. Lett., 28, 4421

Klinglesmith, M. 1997, "The Polar Solar Wind from 2.5 to 40 Solar Radii:

Results of Intensity Scintillation Measurements," PhD Thesis, Univ.of Calif., San Diego

Kohl, J. L. et al 1998, ApJL, 501, 127

Kraichnan, R. H. 1965, Phys. Fluids, 8, 1385

Leamon, R. J., Smith, C. W., Ness, N. F., \& Wong, H. K. 1999, J. Geophys. Res., 104, 22331

Lithwick, Y., \& Goldreich, P. 2001, ApJ, 562, 279

Lithwick, Y., \& Goldreich, P. 2003, ApJ, 582, 1220

Lithwick, Y., Goldreich, P., \& Sridhar, S. 2007, ApJ, 655, 269

Little, L. T., \& Ekers, R. D. 1971, A\&A, 10, 306

Markovskii, S. A. 2004, ApJ, 609, 1112

Markovskii, S. A., \& Hollweg, J. V. 2002a, Geophys. Res. Lett., 29, 24

Markovskii, S. A., \& Hollweg, J. V. 2002b, J. Geophys. Res., 107, 21

Markovskii, S. A., Vasquez, B. J., Smith, C. W., \& Hollweg, J. V. 2006, ApJ, 639, 1177

Marsch, E. 1991, in Physics of the Inner Heliosphere, ed. Schwen, R. \& Marsch, E. (New York: Springer-Verlag: p. 159)

Marsch, E., \& Tu, C.-Y. 1997, A\&A, 319, L17

Marsch, E., Ao, X.-Z., \& Tu, C.-Y. 2004, J. Geophys. Res., 109, 4102

Maron, J. \& Goldreich, P. 2001, ApJ, 554, 1175

Matthaeus, W. H., Zank, G. P., Leamon, R. J., Smith, C. W., Mullan D. J., \& Oughton, S. 1999, Sp. Sci. Rev., 87, 269

Matthaeus, W. H., Zank, G. P., Smith, C. W., \& Oughton, S. 1999, Physical Review Letters, 82, 3444

Matthaeus, W. H., Mullan, D. J., Dmitruk, P., Milano, L., \& Oughton, S. 2003, Nonl. Proc. Geophys., 10, 93

McKenzie, J. F., Ip, W. H., \& Axford, W. I. 1979, Astrophys. Sp. Sci., 64, 183

Newbury, J. A., Russell, C. T., Phillips, J. L., \& Gary, S. P. 1998, J. Geophys. Res., 103, 9553

Parker, E. 1965, Space Sci. Rev., 4, 666

Perez, J., \& Boldyrev, S. 2009, Phys. Rev. Lett., 102, 025003

Podesta, J., \& Bhattacharjee, A., Phys. Rev. Lett., submitted

Quataert, E. 1998, ApJ, 500, 978

Quataert, E., \& Gruzinov, A. 1999, ApJ, 520, 248

Roberts, D. A., Goldstein, M. L., Klein, L. W., \& Matthaeus, W. H. 1987, J. Geophys. Res., 92, 12023

Ruzmaikin, A. \& Berger, M. A. 1998, A\&A, 337, L9

Schekochihin, A. A., Cowley, S. C., Dorland, W., Hammett, G. W., Howes, G. G., Quataert, E., \& Tatsuno, T. 2009, ApJS, 182, 310

Sahraoui, F., Goldstein, M. L., Robert, P., \& Khotyaintsev, Y. V. 2009, Physical Review Letters, 102, 231102

Schwartz, S. J., W. C. Feldman, \& Gary, S. P. 1981, J. Geophys. Res., 86, 541 Scudder, J. D. 1992a, ApJ, 398, 299

Scudder, J. D. 1992b, ApJ, 398, 319

Shebalin, J. , Matthaeus, W., \& Montgomery, D. 1983, J. Plasma Phys., 29, 525

Tu, C. Y., \& Marsch, E., 1990, J. Geophys. Res., 95, 4337

Tu, C. Y., \& Marsch, E., 1995, Sp. Sci. Rev., 73, 1

Tu, C.-Y., \& Marsch, E. 1997, Solar Phys., 171, 363

Tu, C.-Y., \& Marsch, E. 2001a, J. Geophys. Res., 106, 8233

Tu, C.-Y., \& Marsch, E. 2001b, A\&A, 368, 1071

Tu, C.-Y., Pu, Z.-Y., \& Wei, F.-S. 1984, J. Geophys. Res., 89, 9695

Verdini, A., \& Velli, M. 2007, ApJ, 662, 669

Velli, M. 1993, A\&A, 270, 304

Voitenko, Y. \& Goossens, M. 2004, ApJL, 2004, 605, 149

Zhou, Y., \& Matthaeus, W. H. 1990, J. Geophys. Res., 95, 14881 